\title{
Nonlinear Absorption and Self-Phase Modulation in Silicon Optical Fibres
}

\author{
P. Mehta ${ }^{1}$, N. Healy ${ }^{1}$, J.R. Sparks ${ }^{2}$, T.D. Day ${ }^{2}$, P.J.A. Sazio ${ }^{1}$, J.V. Badding ${ }^{2}$, and A.C. Peacock ${ }^{1}$ \\ 1. Optoelectronics Research Centre, University of Southampton, Southampton SO17 1BJ, United Kingdom \\ 2. Department of Chemistry and Materials Research Institute, Pennsylvania State University 16802 PA, USA
}

Silicon optical fibres are generating much interest as a means to directly integrate semiconductor functionality within the fibre architecture to provide a platform for compact all optical signal processing. For example, the high optical nonlinearity of the core material opens up the potential for these fibres to be used for signal regeneration in very short device lengths. In this paper we characterise the nonlinear optical properties of a step index hydrogenated amorphous silicon (a-Si:H) optical fibre and demonstrate its use for broadband self-phase modulation (SPM). The fibre was fabricated using a high pressure chemical fluid technique [1] to deposit the semiconductor into the central hole of a silica capillary. The resulting fibre had a core diameter of $6 \mu \mathrm{m}$ and a length of $1.5 \mathrm{~cm}$, with the linear transmission losses measured to be as low as $1.7 \mathrm{~dB} / \mathrm{cm}$ at $1550 \mathrm{~nm}$. As the effective nonlinear interaction length is reduced by the material loss, the realization of low losses is a necessary requirement for the observation of these processes.

To characterize the nonlinear properties we compare transmission measurements with numerical modelling of the pulse evolution in the fiber to obtain estimates for the carrier lifetime, two-photon absorption (TPA) $\beta_{\text {TPA }}$, freecarrier absorption (FCA) $\sigma_{\mathrm{FCA}}$, and the Kerr nonlinearity $n_{2}$. All measurements are conducted using $650 \mathrm{fs}$ pump pulses generated via a $40 \mathrm{MHz}$ fibre laser, operating at $1540 \mathrm{~nm}$. To estimate the carrier lifetime, we first conduct a pump-probe experiment based on cross-absorption modulation (XAM) [2] using a low power CW probe at $1570 \mathrm{~nm}$. By fitting the results shown in the inset of Fig. 1(a), we obtain a relatively long lifetime of $\tau \sim 80 \mathrm{~ns}$ which we attribute to the high material quality (i.e., minimal defect sites) and the large cross-section of our silicon core (i.e., fewer surface states per volume). The nonlinear absorption as a function of coupled peak power is shown in Fig. 1(a). The fitted curve is obtained using a simplified time domain propagation model which, using the measured value of $\tau$, yields $\beta_{\mathrm{TPA}} \sim 0.8 \mathrm{~cm} / \mathrm{GW}$ and $\sigma_{\mathrm{FCA}} \sim 1 \times 10^{-16} \mathrm{~cm}^{2}$, in agreement with previous reports for on-chip silicon waveguides $[2,3]$. The spectral broadening of the pulses is then shown in Fig. 1(b), clearly illustrating the extent of the frequency generation at high pump powers. Comparison with modelling of the nonlinear Schrödinger equation reveals a value of $n_{2} \sim 1.8 \times 10^{-13} \mathrm{~cm}^{2} / \mathrm{W}$, which is greater than twice that of crystalline silicon [3]. The potential for these silicon optical fibres to be used in ultrafast all-optical processing will be discussed.
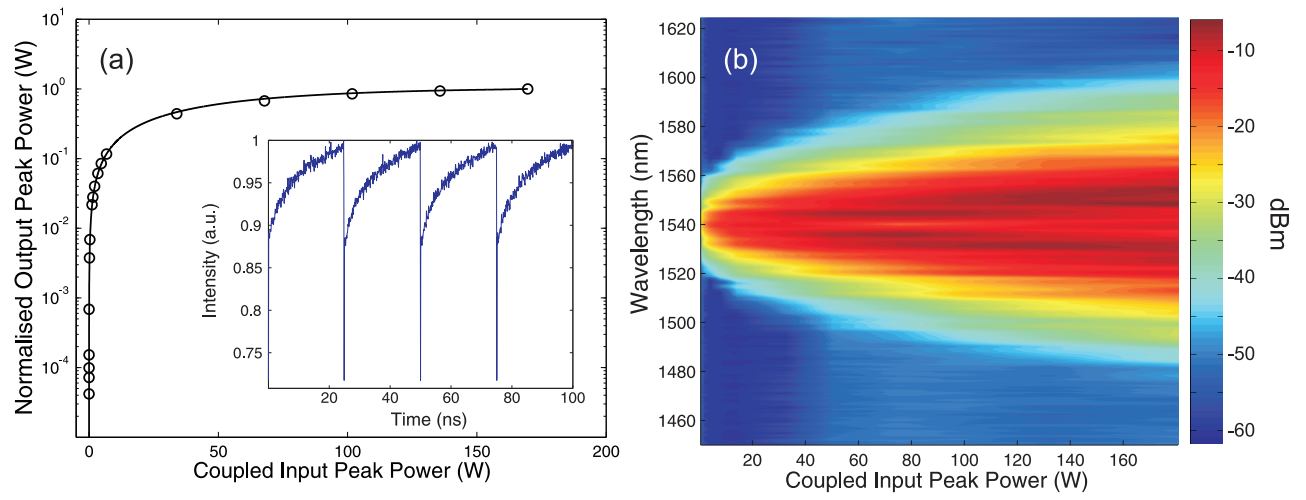

Fig. 1.(a) Nonlinear absorption measurements (circles) with a simulated fit of the temporal pulse propagation (solid line). Inset shows carrier recombination following XAM. (b) Broadband self-phase modulation.

\section{References}

[1] P. J. A. Sazio, A. Amezcua-Correa, C. E. Finlayson, J. R. Hayes, T. J. Scheidemantel, N. F. Baril, B. R. Jackson, D. J. Won, F. Zhang, E. R. Margine, V. Gopalan, V. H. Crespi, and J. V. Badding, "Microstructured optical fibers as high-pressure microfluidic reactors," Science 311, 1583-1586 (2006).

[2] K. Narayanan, A. W. Elshaari, and S. F. Preble, "Broadband all-optical modulation in hydrogenated-amorphous silicon waveguides," Opt. Express 18, 9809-9814 (2010).

[3] H. K. Tsang and Y. Liu, "Nonlinear optical properties of silicon waveguides," Semicond. Sci. Technol. 6, 064007 (2008). 